\title{
Accurate Target Tracking: A New Kalman Filter Residue-Based Approach Applied To a Nonlinear Multivariable Control System
}

\author{
Rajamani Doraiswami ${ }^{1}$, Lahouari Cheded ${ }^{2}$, and Sreeraman Rajan ${ }^{3}$ \\ ${ }^{1}$ Department of Electrical and Computer Engineering, University of New Brunswick, Fredericton \\ New Brunswick, Canada dorai@ unb.ca \\ ${ }^{2}$ Professor.Independent Researcher and Consultant, UK. Life SMIEEE cheded@kfupm.edu.sa \\ ${ }^{3}$ Systems and Computer Science, Carleton University, Ontario, Canada sreeramanr@ sce.carleton.sa
}

\begin{abstract}
A nonlinear system is approximated using the linear parameter-varying (LPV) model described by piecewise-linear BoxJenkins (BJ) model at each operating point. Both the input and the output measurements are corrupted by unknown stochastic disturbance and measurement noise. A data-based approach is used to extract the true input from the corrupted input measurement. The system and its associated Kalman filter(KF) are identified, without any a-priori knowledge of the disturbance and measurement noise statistics, by using emulator-generated data by minimizing the KF residual so that identified models are robust to model perturbations, accurate, consistent, and reliable. A fault-tolerant controller is thus developed. An adaptive filter, which is a series combination of the inverse of the signal model and the desired signal model, is driven by the signal estimate and the residual, ensuring that its ouput tracks the desired signal despite model perturbations and without any cumbersome controller redesign. An internal model-based robust state-feedback controller is proposed to accurately track a desired trajectory despite model perturbations and stochastic disturbances. The internal model is driven by the error between the reference input and the adaptive filter output. The proposed scheme was successfully evaluated on a simulated autonomously-guided aerial vehicle.
\end{abstract}

Keywords: nonlinear system, Box-Jenkins model, identification, Kalman filter, adaptive filter

\section{Introduction}

A KF-based state feedback robust controller is proposed for a Multiple-Input and Multiple-Output (MIMO) nonlinear system such as terrestrial and aerial vehicles for accurately tracking a desired path in $\mathrm{x}, \mathrm{y}$ and $\mathrm{z}$ directions despite model perturbations and unknown stochastic disturbance and measurement noise corrupting both the input and the output [1-3]. The model whose input and output are corrupted is termed as an errors-in variables one [4-5]. As the analysis, design, estimation, identification, and control of nonlinear system are not mathematically tractable in a true nonlinear setting, a linear parameter-varying (LPV) model, is used to approximate the nonlinear system [6]. The resulting piecewise-linear models help develop computationally simple, efficient, and robust schemes for identification, design of $\mathrm{KF}$, fault detection and isolation, fault tolerance and condition-based maintenance.

Predictive analytics is of paramount importance in resolving a host of problems in a variety of fields including engineering, financial and medical and relies on a wide range of tools such as statistical modelling and analytical techniques, machine learning, artificial intelligence, and deep learning algorithms [7-8]. The true input is estimated from the corrupted input by exploiting the smoothness of the true input and the randomness of the corrupted input using two-stage frequency domain identification [8]. For notational convenience, the estimated true input is termed henceforth merely as input. The output error defined as the sum of disturbance and measurement noise and is assumed to be bounded. The signal and the disturbance are both modelled as outputs of linear time-invariant systems driven by the input signal, and a Gaussian zero-mean white noise process, respectively. It is assumed that the signal, disturbance, and measurement noise are all uncorrelated with each other.

A piecewise-linear Box-Jenkins (BJ) MIMO system relating the input and corrupted output is obtained as the best approximation to the nonlinear model. The BJ model of the system is an augmented model of the signal and the disturbance models [9-10]. This model covers several imporatant practical systems, including for example, autonomous vehicles and autonomously-guided drones flying at different altitudes and subject to varying wind gusts that may accelerate or decelerate, push or pull the drone away from the desired trajectory. These devices are currently enjoying an ever-increasing field of 
important applications. A state feedback controller is designed using the internal model principle to ensure an accurate tracking of the reference waveform, despite the presence of model perturbations caused by a wide class of unavoidable stochastic disturbances, and of the measurement noise corrupting the signal [11]. The objective is to ensure that the output track accurately the desired reference trajectory. Using inverse dynamics, the desired input is estimated. The proposed controller is designed and implemented using the KF estimates of the signal, the states, the output error and the disturbance model estimate. A state-feedback controller based on the KF-based state feedback must meet the stability and performance requirements without the need for continuous adaptation when the plant is perturbed in the neighborhood of the nominal operating point generated by the emulator-perturbed experiments.

The feedback controller is implemented as follows:

- An internal model of the reference is included in the controller. Note that an internal model of a signal is a transfer matrix whose zero-input response is a copy of the signal. The denominator of the internal model and that of the signal are identical to each other.

- The internal model is driven by the tracking error, which is error between the reference trajectory and the estimate of the signal, rather than that of the estimate of the output, which is the signal corrupted by the disturbance and measurement noise

- The closed-loop system is asymptotically stable despite model perturbations

- The state feedback law is implemented using the KF state estimate, as per the separation principle-based design strategy.

Unlike in the conventional approach, it is unnecessary to include the internal model of the disturbance as the KF separates the estimate of the signal from that of the output which is formed of the signal, the disturbance and measurement noise. Further, it is not necessary here to employ the feedforward of the measured disturbance.

In system identification, the criterion for determining whether the identified model has captured completely the static and dynamic behaviors of the system is that the residual of the KF associated with the system be a zero-mean white noise process [9-10]. If the equation error is a zero-mean white noise process, the estimate will be unbiased and efficient; otherwise, it will be biased. In view of the above-stated key properties, the residual then becomes a zero-mean white noise process if and only if the system and the identified model embodied in the KF are identical. The identification objective of ensuring the residual is a zero-mean white noise process, will ensure not only that the KF is accurately identified but also that the Kalman gain is optimal, thereby avoiding the need to specify the covariance of the disturbance and the measurement noise and to use the Riccati equation to solve for the Kalman gain.

Novel Emulators: The static and dynamic behaviors of a physical system change as a result of variations in the parameters of some of its subsystems such as sensors, actuators, plant, disturbance models and controllers. As the parameters of these subsystems are not generally accessible to generate data, instead, novel emulators which are product of first-order all-pass are used, are connected in cascade with the output, input or both, of the subsystems. An emulator mimics the variations in the associated subsystems including the disturbance model, some of which have not been seen by the system, thus enriching the data set used for both identification and control purposes. An emulator induce gain or phase variations in the subsystem it is connect to. Emulator parameters are perturbed to mimic various normal and abnormal, or faulty, operating scenarios resulting from variations in these subsystems. The emulator-generated data is employed in a) the identification of robust systems and signal models and their associated KFs using the two-stage identification scheme, and b) the design of robust controllers the robust system model and the associated KF are identified using the emulator-generated data; and the signal and the output error are estimated. From the identified models, the signal model and the associated KF are identified. Physical systems are invariably subject to model perturbations. In the absence of model perturbations, the controller tracks accurately the desired reference waveform ensuring that the tracking error is asymptotically zero. Thanks to identification using emulator-generated data, the identified models are robustly stable. However, the performance of the controller may not be robust to model perturbations as the rise time, overshoot and settling time may vary. To overcome this problem, the residual of KF estimate of the signal is adapted to these variations. If the input to the KF is an ideal unperturbed signal, then its residual will be zero in finite time. On the other hand, with a perturbed input the residual will be zero asymptotically. An adaptive signal is obtained using the KF residual and the input signal so that the adaptive filter output is the ideal unperturbed 
signal. As such, the adaptive filter output will be the ideal signal independent of whether the input signal is perturbed or not, this ensuring robust performance. The computationally-burdensome controller redesign that would be needed in a conventioanl LQR approach is ths avoided. The adaptation strategy plays the role of a 'model-reference' adaptive controller without an online controller adaptation.

The proposed scheme finds applications in many areas of control theory and in diverse applications including robust control of autonomous vehicles to track accurately a desired trajectory using the signal estimated from the output corrupted by disturbance and measurement noise, and not from the noisy estimate of the output. The motivation of the work stems primarily from the need to detect accurately and reliably leakage in pipelines using drones that are controlled to specifically track a desired trajectory that represents the pipeline track to be monitored with a view to collecting data for specific applications. By way of example, the desired trajectory could be a long pipeline running in a dangerous and/or environmentally-unfriendly area, and collecting data from sensors placed at some suitable locations along the pipeline, in order to timely detect possible leaks in the pipeline

The proposed feedback controller scheme is evaluated on a simplified simulated model of a drone moving in a horizontal plane to track accurately the desired trajectory in spite of wind gusts and model perturbations.

Main contributions: To the best of the authors' knowledge, the contributions include the following:

a) The key theoretical contribution lies in using the KF to estimate the signal component from the output formed of an additive sum of the signal, the stochastic disturbance and measurement noise.

b) Rigorous mathematical foundations for the proposed scheme are given. It is shown that the following key properties of the KF hold if and only if the identified model embodied in the KF is identical to the system model

- The residual model of the KF maps the system input and output to the KF residual. The signal model is a matrix fraction description relating the system input and output of the residual model

- The two KFs, one associated with the system and the other with the signal model, are employed to develop faulttolerant and condition-based maintenance systems. The residuals of the KFs of the system and the signal monitor the status of the overall system and the signal model, respectively, thereby isolating a fault from other variations in the output error and thus lowering the false alarm probability. A fault is asserted only if the residuals of the KFs for both the system and especially the signal vary.

c) The use of the powerful and novel concept of emulators which are connected to the subsystem's input, or output or to both to mimic likely operating scenarios, such as normal and abnormal or faulty operating scenarios, including variations in the disturbance and measurement noise, that have not been seen by the system. The emulator parameters are varied to generate a set of emulator-perturbed input-output data and a set of perturbed models

d) Using emulator-generated input-output data, a two-stage identification scheme is proposed using the key property of the KF for obtaining robust and accurate models of the system and the signal, and their associated KFs.

e) A novel robust combined feedforward-feedback controller is implemented using the internal model principle that ensures accurate tracking of the reference trajectory despite the presence of stochastic disturbances and measurement noise. The feedforward controller is implemented without the need for a separate measurement of the disturbance because the feedback controller needs only the internal model of the reference and not that of the disturbance.

f) A novel adaptive filer scheme is developed to ensure robust performance

\section{System Model}

\subsection{Mathematical Model of The System}

The Multiple-Input and Multiple-Output (MIMO) nonlinear state-space model of a system is: 


$$
\begin{aligned}
& \boldsymbol{x}(k+1)=\boldsymbol{f}\left(\boldsymbol{x}(k), \boldsymbol{u}(k), \boldsymbol{u}_{w}(k)\right) \\
& \boldsymbol{y}(k)=\boldsymbol{g}(\boldsymbol{x}(k), v(k))
\end{aligned}
$$

where $\boldsymbol{x}(k) \in R^{n}, \boldsymbol{u}(k) \in R^{p}$ is the scalar control input, $\boldsymbol{y}(k) \in R^{q}$ is a vector formed of all measured (accessible) outputs, $\boldsymbol{v}(k) \in R^{q}$ is a measurement noise and $\boldsymbol{u}_{w}(k) \in R^{p}$ is an inaccessible input that generates the stochastic disturbance $\boldsymbol{w}(k) \in R^{q}$ , $\boldsymbol{f}(.) \in R^{n}, \boldsymbol{g}(.) \in R^{q}$ are nonlinear smooth and differentiable functions. The output $\boldsymbol{y}(k)$ is the output that is corrupted by disturbance and measurement noise.

\subsection{Linear Parameter-Varying (Lpv) Model: 2.2.1 Input Measurement Model}

The measurement input $\boldsymbol{u}(k) \in R^{p}$ is a sum of the unknown true input $\boldsymbol{u}_{0}(k)$ and the combined disturbance and measurement noise $\boldsymbol{u}_{v}(k)$ :

$$
\boldsymbol{u}(k)=\boldsymbol{u}_{v}(k)+\boldsymbol{u}_{0}(k)
$$

Let the state space model $\left(\boldsymbol{A}_{v}, \boldsymbol{B}_{v}, \boldsymbol{C}_{v}\right)$ of the corrupting input $\boldsymbol{u}_{v}(k)$ be:

$$
\begin{aligned}
& \boldsymbol{x}_{v}(k+1)=\boldsymbol{A}_{v} \boldsymbol{x}_{v}(k)+\boldsymbol{B}_{v} \boldsymbol{v}_{1}(k) \\
& \boldsymbol{u}_{v}(k)=\boldsymbol{C}_{v} \boldsymbol{x}_{v}(k)+\boldsymbol{v}_{2}(k)
\end{aligned}
$$

Predictively analytics: The inaccessible true input $\boldsymbol{u}_{0}(k)$ is assumed to be a smooth input such as a sum of sinusoids while the noisy $\boldsymbol{u}_{v}(k)$ exhibits random fluctuation. The inaccessible $\boldsymbol{u}_{0}(k)$ is extracted from $\boldsymbol{u}(k)$ using the frequency-domain approach by exploiting their spectral characteristics such as the smooth waveform $\boldsymbol{u}_{0}(k)$ has line-spectra, and noisy $\boldsymbol{u}_{v}(k)$ has wildly-varying spectra. The estimated true input, is derived from the accessible input $u(k)$ using a predictive analytics approach, involving an artificial intelligence or machine learning algorithm [8-10]. The estimated true denoted simply as input replaces the true input $\boldsymbol{u}_{0}(t)$ in the rest of this paper. As a result, the system model relates the true input and the corrupted output $\boldsymbol{y}(k)$, which is a sum of the signal $\boldsymbol{s}(k)$, the output disturbance $\boldsymbol{w}(k)$, and the measurement noise $\boldsymbol{v}(k)$ :

$$
\boldsymbol{y}(z)=\boldsymbol{s}(z)+\boldsymbol{w}(z)+\boldsymbol{v}(z)=\boldsymbol{s}(z)+\vartheta(z)
$$

Where $\vartheta(k)$ is the output error;

The state-space model of the signal $s(k) \in R^{q},\left(\boldsymbol{A}_{s}, \boldsymbol{B}_{s}, \boldsymbol{C}_{s}\right)$, is;

$$
\begin{aligned}
& \boldsymbol{x}_{s}(k+1)=\boldsymbol{A}_{s} \boldsymbol{x}_{s}(k)+\boldsymbol{B}_{s} \boldsymbol{u}(k) \\
& \boldsymbol{s}(k)=\boldsymbol{C}_{s} \boldsymbol{x}_{s}(k)
\end{aligned}
$$

The state-space model of the disturbance $\boldsymbol{w}(k) \in R^{q},\left(\boldsymbol{A}_{w}, \boldsymbol{B}_{w}, \boldsymbol{C}_{w}\right)$, is;

$$
\begin{aligned}
& \boldsymbol{x}_{w}(k+1)=\boldsymbol{A}_{w} \boldsymbol{x}_{w}(k)+\boldsymbol{B}_{w} \boldsymbol{u}_{w}(k) \\
& \boldsymbol{w}(k)=\boldsymbol{C}_{w} \boldsymbol{x}_{w}(k)
\end{aligned}
$$

Where $\boldsymbol{x}_{s} \in R^{n_{s}} ; \boldsymbol{x}_{w} \in R^{n_{w}} ; \boldsymbol{A}_{s} \in R^{n_{s} x n_{s}} ; \boldsymbol{A}_{w} \in R^{n_{w} x n_{w}}, \boldsymbol{B}_{s} \in R^{n_{s} x p} ; \boldsymbol{B}_{w} \in R^{n_{w} x p} ; \boldsymbol{C}_{s} \in R^{q x n_{s}} ; \boldsymbol{C}_{w} \in R^{q x n_{w}} ; \boldsymbol{u}_{w} \in R^{p} ; n_{s}$ and $n_{w}$ are respectively the orders of the signal $s(k)$ and disturbance models;

\section{Assumptions}

- The signal model $\left(\boldsymbol{A}_{s}, \boldsymbol{B}_{s}, \boldsymbol{C}_{s}\right)$ and disturbance model $\left(\boldsymbol{A}_{w}, \boldsymbol{B}_{w}, \boldsymbol{C}_{w}\right)$ are controllable and observable 
- The disturbance model $\left(\boldsymbol{A}_{w}, \boldsymbol{B}_{w}, \boldsymbol{C}_{w}\right)$ is asymptotically stable

- The input $\boldsymbol{u}_{w}(k)$ driving the disturbance model is zero mean white Gaussian process

- The measurement noise $\boldsymbol{v}(k)$ is zero mean white Gaussian process

- The signal $\boldsymbol{s}(k)$, the disturbance $\boldsymbol{w}(k)$ and measurement noise $\boldsymbol{v}(k)$ are uncorrelated

\subsubsection{State-SPACE Box-Jenkins MODEL}

The linearized (MIMO) state-space model $(\boldsymbol{A}, \boldsymbol{B}, \boldsymbol{C})$ at an operating point $\xi_{i}$ is an augmented model of the signal and output disturbance given by:

$$
\begin{aligned}
& \boldsymbol{x}(k+1)=\boldsymbol{A} \boldsymbol{x}(k)+\boldsymbol{B u}_{u, u_{w}}(k) \\
& \boldsymbol{y}(k)=\boldsymbol{C} \boldsymbol{x}(k)+\boldsymbol{v}(k)
\end{aligned}
$$

where $\boldsymbol{A} \in R^{n x n}, \boldsymbol{B} \in R^{n x 2 p}, \boldsymbol{C} \in R^{q x n} ; \boldsymbol{x}(k) \in R^{n}$ is the state; $\boldsymbol{u}_{u, u_{w}}=\left(\boldsymbol{u}(k), \boldsymbol{u}_{w}(k)\right)^{T} \in R^{2 p}$ is a augmented input formed of input $\boldsymbol{u}_{w}(k) \in R^{q}$ and $\boldsymbol{u}_{w}(k) \in R^{q}$ is a zero-mean Gaussian white noise process that is inaccessible; $\boldsymbol{v}(k) \in R^{q}$ is a measurement noise that is a zero-mean Gaussian white noise process; $\boldsymbol{A}=\left[\begin{array}{cc}\boldsymbol{A}_{s} & 0 \\ 0 & \boldsymbol{A}_{w}\end{array}\right] ; \boldsymbol{B}=\left[\begin{array}{cc}\boldsymbol{B} & 0 \\ 0 & \boldsymbol{B}_{w}\end{array}\right] ; \boldsymbol{C}=\left[\begin{array}{ll}\boldsymbol{C}_{s} & \boldsymbol{C}_{w}\end{array}\right]$;

\section{Controller Design}

The measurement output $\boldsymbol{y}(k)$ (4) is assumed to be an additive combination of the signal $s(k)$, the stochastic disturbance $\boldsymbol{w}(k)$ and the measurement noise $\boldsymbol{v}(k)$.

\subsection{Internal Model-Based Controller}

- Using inverse dynamics, the desired input $\boldsymbol{u}_{d}(k)$ is estimated so end effector tracks the desired output trajectory $\boldsymbol{y}_{d}(k)$. The proposed controller is designed and implemented using the KF estimates

- The signal models of systems such as aerial vehicles, and quadrotor are generally unstable, and need to be pre-

- stabilized so that the system may be reliably identified. A state feedback controller is designed using the internal model principle to ensure an accurate tracking of the reference waveform, despite the presence of model perturbations caused by a wide class of stochastic disturbances, and measurement noise corrupting the signal.

- An optimal state-feedback controller for the Box-Jenkins model is determined assuming the states of the signal model are available. The assumed states of the signal model are substituted by those estimated by the associated Kalman filter. The feedback control input to the signal model becomes in Fig. 2:

$$
\hat{\boldsymbol{u}}(k)=-\boldsymbol{F}_{s} \hat{\boldsymbol{x}}_{s}(k)-\boldsymbol{F}_{I} \boldsymbol{x}_{I}(k)
$$

- The resulting closed loop Box-Jenkins model $\left(\boldsymbol{A}_{c l}, \boldsymbol{B}_{c l}, \boldsymbol{C}_{c l}\right)$ given by:

$$
\begin{aligned}
& \boldsymbol{x}(k+1)=\boldsymbol{A}_{c l} \boldsymbol{x}(k)+\boldsymbol{B}_{c l} \boldsymbol{r}(k) \\
& \boldsymbol{y}(k)=\boldsymbol{C}_{c l} \boldsymbol{x}(k)+\boldsymbol{v}(k)
\end{aligned}
$$

- Where; $\boldsymbol{x}=\left[\begin{array}{c}\boldsymbol{x}_{s} \\ \boldsymbol{x}_{i m} \\ \boldsymbol{x}_{w}\end{array}\right] \in R^{n} ; \boldsymbol{A}_{c l}=\left[\begin{array}{ccc}\boldsymbol{A}_{s}-\boldsymbol{B}_{s} \boldsymbol{F}_{s} & -\boldsymbol{B}_{s} \boldsymbol{F}_{I} & 0 \\ -\boldsymbol{B}_{I} \boldsymbol{C}_{c l} & \boldsymbol{A}_{I} & 0 \\ 0 & 0 & \boldsymbol{A}_{w}\end{array}\right] \in R^{n x n} ; \boldsymbol{B}=\left[\begin{array}{ccc}\boldsymbol{B}_{s} & 0 & 0 \\ 0 & \boldsymbol{B}_{I} & 0 \\ 0 & 0 & \boldsymbol{B}_{w}\end{array}\right] ; n=n_{c l}+n_{w}$ is the order of the system; $\boldsymbol{r}(k)=\left(0,\left(\boldsymbol{s}(k)-\boldsymbol{u}_{d}(k)\right), \boldsymbol{u}_{w}(k)\right)^{T} \in R^{n}, \boldsymbol{C}=\left[\begin{array}{lll}\boldsymbol{C}_{s} & \boldsymbol{C}_{I} & \boldsymbol{C}_{w}\end{array}\right] \in \square^{q x n}$ is the output vector; 


\section{Novel Accurate Emulator-Based Identification}

An accurate emulator-based model identification scheme is proposed and employed here. An emulator, which is modelled as a product of first-order all-pass filters and which induces phase and gain changes, is connected in cascade to the input, output or both, of the signal model to emulate a set of likely operating regimes around the nominal operating point. The identified model is obtained as the best fit over all emulated operating regions, thereby ensuring both accuracy and robustness of the identified model. A high-performance robust controller is designed for the identified model using a mixed sensitivity $H_{\infty}$ approach.

- In the first stage, a robust model of the system fault-free nominal system $\left(\boldsymbol{A}^{0}, \boldsymbol{B}^{0}, \boldsymbol{C}^{0}\right)$ and its associated Kalman filter $\left(\boldsymbol{A}^{0}-\boldsymbol{K}^{0} \boldsymbol{C}^{0},\left[\boldsymbol{B}^{0} \boldsymbol{K}^{0}\right], \boldsymbol{C}^{0}\right)$ are identified from the set of the emulator-generated input-output data using the structure of the residual model of the Kalman filter. Then the estimate $s^{0}(k)$ of the signal $s(k)$ and the estimate $\hat{\vartheta}(k)$ of the output error $\vartheta(k)$ and the disturbance model are derived.

- In the second stage, using the key properties established the signal model $\left(\boldsymbol{A}_{s}^{0}, \boldsymbol{B}_{s}^{0}, \boldsymbol{C}_{s}^{0}\right)$ and its associated Kalman filter $\left(\boldsymbol{A}_{s}^{0}-\boldsymbol{K}_{s}^{0} \boldsymbol{C}_{s}^{0},\left[\boldsymbol{B}_{s}^{0} \boldsymbol{K}_{s}^{0}\right], \boldsymbol{C}_{s}^{0}\right)$ are obtained

\section{Kalman Filter: Properties, Design and Applications to Status Monitoring}

We shall now state some vital KF properties that help design the required KFs in an accurate and robust way. We shall also discuss the KF application of status monitoring that is so vital to many industrial areas.

\subsection{KF Properties}

\subsubsection{Residual model}

The frequency-domain expression relating the input $\hat{\boldsymbol{u}}(z) \in R^{p}$ and the output $\boldsymbol{y}(z) \in R^{q}$ to the residual $\boldsymbol{e}(z) \in R^{q}$ is given by the following model termed the residual model:

$$
\boldsymbol{e}(z)=F^{-1}(z) \overline{\boldsymbol{D}}(z) \boldsymbol{y}(z)-F^{-1}(z) \overline{\boldsymbol{N}}(z) \boldsymbol{u}(z)
$$

where $\overline{\boldsymbol{D}}(z)$ and $\overline{\boldsymbol{N}}(z)$ are matrix polynomials, $F(z)$ is the scalar characteristic polynomial termed Kalman polynomial, $F(z)=\operatorname{det}\left(z \boldsymbol{I}-\boldsymbol{A}^{0}+\boldsymbol{K}^{0} \boldsymbol{C}^{0}\right) ; D(z)=\operatorname{det}\left(z \boldsymbol{I}-\boldsymbol{A}^{0}+\boldsymbol{K}^{0} \boldsymbol{C}^{0}\right) ; \overline{\boldsymbol{D}}(z)=F(z)\left(\boldsymbol{I}-\boldsymbol{C}^{0}\left(z \boldsymbol{I}-\boldsymbol{A}^{0}+\boldsymbol{K}^{0} \boldsymbol{C}^{0}\right)^{-1} \boldsymbol{K}^{0}\right)$ is a $q x q$ matrix; $\overline{\boldsymbol{N}}(z)=F(z)\left(\boldsymbol{C}^{0}\left(z \boldsymbol{I}-\boldsymbol{A}^{0}+\boldsymbol{K}^{0} \boldsymbol{C}^{0}\right)^{-1} \boldsymbol{B}^{0}\right)$ is qxp matrix; $\boldsymbol{I} \in R^{q x q}$ is an identity matrix. The rational polynomials $F^{-1}(z) \overline{\boldsymbol{D}}(z)$ and $F^{-1}(z) \bar{N}(z)$ are associated with the system output $\boldsymbol{y}(z)$ and the input $\hat{\boldsymbol{u}}(z)$., respectively

Remarks: The residual model of the KF forms the backbone of the proposed identification scheme.

\subsubsection{Key KF properties}

These are fully established in [10-11] and are restated herein for clarification.

Lemma 2: The residual is a zero-mean white noise process with minimum variance if and only if the model of the system, denoted $(\boldsymbol{A}, \boldsymbol{B}, \boldsymbol{C})$ and the identified model embodied in the KF, denoted $\left(\boldsymbol{A}^{0}, \boldsymbol{B}^{0}, \boldsymbol{C}^{0}\right)$ are identical.

$$
(\boldsymbol{A}, \boldsymbol{B}, \boldsymbol{C})=\left(\boldsymbol{A}^{0}, \boldsymbol{B}^{0}, \boldsymbol{C}^{0}\right)
$$

Corollary: If $(\boldsymbol{A}, \boldsymbol{B}, \boldsymbol{C}) \neq\left(\boldsymbol{A}^{0}, \boldsymbol{B}^{0}, \boldsymbol{C}^{0}\right)$, the residual will not be a zero-mean white noise process as there will be an additive term, $\boldsymbol{e}_{f}(k)$ referred to as a fault indicative term:

$$
\boldsymbol{e}(k)=\boldsymbol{e}_{f}(k)+\boldsymbol{e}_{0}(k)
$$

Where $\boldsymbol{e}_{0}(k)$ a zero-mean white noise is process; 
The fault indicator term in frequency domain $\boldsymbol{e}_{f}(z)$, represent perturbations coming from the system model $\Delta \boldsymbol{G}(z)$, or the stochastic disturbance model $\boldsymbol{G}_{w}(z)$ or both. The size of the fault is determined by the norm of fault indication term $\left\|\boldsymbol{e}_{f}(z)\right\|$; The fault diagnosis scheme is developed using the fault indicator term forms $\boldsymbol{e}_{f}(z)$.

The following Lemmas are developed by assuming that Lemma 1 holds.

Derivation of the signal and its model: The following lemma 3 shows that the estimates of the signal and its model may be derived from the residual modelError! Reference source not found.

\section{Lemma 3:}

The estimates of the signal model, the signal and the output error are:

$$
\begin{aligned}
& \hat{\boldsymbol{G}}(z)=\frac{\overline{\boldsymbol{N}}(z) D_{w}(z)}{\bar{D}(z) D_{w}(z)}=\frac{\boldsymbol{N}_{s}(z)}{D_{s}(z)}=\hat{\boldsymbol{G}}_{s}(z) \\
& \hat{\boldsymbol{s}}(z)=\hat{\boldsymbol{G}}_{s}(z) \hat{\boldsymbol{u}}(z) ; \hat{\boldsymbol{\vartheta}}(z)=\boldsymbol{y}(z)-\hat{\boldsymbol{s}}(z)
\end{aligned}
$$

Let the state space model be $\left(\hat{\boldsymbol{A}}_{s}^{0}, \hat{\boldsymbol{B}}_{s}^{0}, \hat{\boldsymbol{C}}_{s}^{0}\right)$ associated with the transfer function $\hat{\boldsymbol{G}}_{s}(z)$

Remark: There are two approaches to identifying the signal model and the signal. One approach is by deriving them from the residual model of the Kalman filter as shown in Lemma 2 given by (12) and the other approach identify system model using the input $\hat{\boldsymbol{u}}$ and the output $\boldsymbol{y}$ and determining its minimal realization:

The identified system model $\hat{\boldsymbol{G}}(z)$ is non-minimal (12) and the signal model $\hat{\boldsymbol{G}}_{s}(z)$ is its minimal realization.

\subsection{Kalman-Filter Design for the Signal:}

The residual model of the KF for the signal model becomes:

$$
\boldsymbol{e}_{k f s}(z)=\frac{\overline{\boldsymbol{D}}_{s}(z)}{\bar{F}_{s}(z)} \hat{\boldsymbol{s}}(z)-\frac{\overline{\boldsymbol{N}}_{s}(z)}{\bar{F}_{s}(z)} \boldsymbol{r}(z)
$$

Lemma 5: The residual $\boldsymbol{e}_{k f s}(k)$ is asymptotical zero if and only if the model of the closed loop system that has generated the input-output data $(\boldsymbol{r}(k), \boldsymbol{s}(k))$, denoted $\left(\boldsymbol{A}_{c l}, \boldsymbol{B}_{c l}, \boldsymbol{C}_{c l}\right)$ and the identified model $\left(\boldsymbol{A}_{c l 0}, \boldsymbol{B}_{c l 0}, \boldsymbol{C}_{c l 0}\right)$ embodied in the KF are identical, that is $\left(\boldsymbol{A}_{c l}, \boldsymbol{B}_{c l}, \boldsymbol{C}_{c l}\right)=\left(\boldsymbol{A}_{c l 0}, \boldsymbol{B}_{c l 0}, \boldsymbol{C}_{c l 0}\right)$ :

$$
\operatorname{limit}_{k \rightarrow \infty}\left\{\boldsymbol{e}_{k f s}(k)\right\}=0
$$

Lemma 6: The residual $\boldsymbol{e}_{k f s}(k)$ is not asymptotical zero if $\left(\boldsymbol{A}_{c l}, \boldsymbol{B}_{c l}, \boldsymbol{C}_{c l}\right) \neq\left(\boldsymbol{A}_{c l 0}, \boldsymbol{B}_{c l 0}, \boldsymbol{C}_{c l 0}\right)$;

$$
\operatorname{limit}_{k \rightarrow \infty} \boldsymbol{e}_{k f s}(k)=\boldsymbol{e}_{f}(k) \neq 0
$$

The KF estimate of the perturbed signal using adaptive filter, $\boldsymbol{s}_{a d p}(k)$ is given by

$$
\boldsymbol{s}_{a d p}(k)=\hat{\boldsymbol{s}}_{k f s}(k)-\boldsymbol{e}_{k f s}(k)
$$

\subsection{Application to Status Monitoring: Conventional And Proposed Approaches}

The residuals $\boldsymbol{e}(k)$ and $\boldsymbol{e}_{k f s}(k)$ of the KFs (11) and are employed to monitor the status of the overall system and to detect and isolate faults in the signal and disturbance models and the sensors. The proposed scheme provides a sound framework for developing fault-tolerant systems as well as condition-based maintenance systems.

The implementation of the KF-based robust combined feedforward-feedback controller shown in Fig 1: 


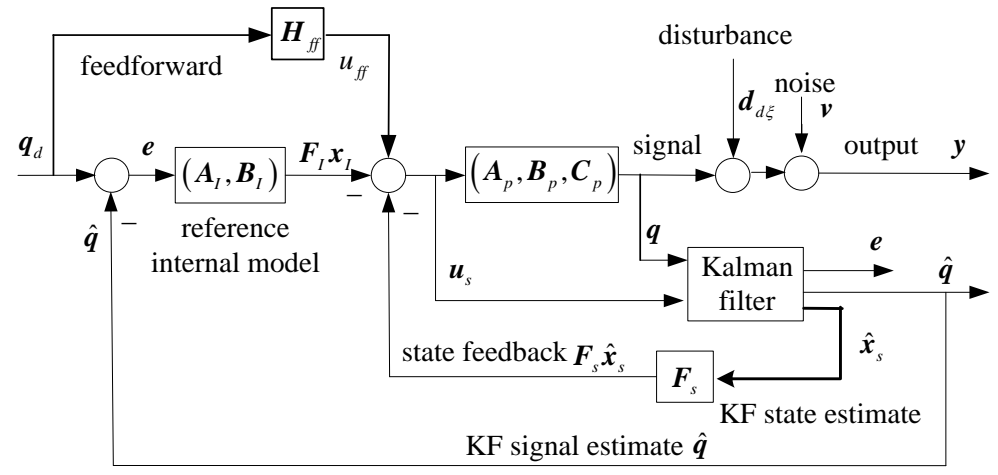

Fig 1: KF-based feedforward-state-feedback controller

Proposed approach: The estimates of the closed-loop signal model, the signal and the states are determined using the key properties of the KF. The disturbance and measurement noise-free KF estimates ate employed instead of their noisy counterparts, namely the noisy output and the noisy states, respectively, for implementing the feedback controller. The tracking error driving the internal model is the error between the desired and the estimated signals, and the state-feedback is implemented using the estimates of the states of the KF. The internal model includes only of the reference as the deterministic disturbance, is unknown

\section{Evaluation of Proposed Scheme on a Simulated Drone \\ 6.1 Illustrative Example with the KF-Based Adaptive Filter}

An illustrative example of tracking a sinusoidal input, $r(k)=\sin (\omega k)$ despite model perturbations using the proposed KF-based adaptive filter.

$$
A_{c l}=\left[\begin{array}{cccc}
-0.5248 & -2.0305 & 1.0531 & -0.8906 \\
0.2376 & -0.0153 & 0.5265 & -0.4453 \\
0 & -1 & 1.9319 & -1 \\
0 & 0 & 1 & 0
\end{array}\right] \in R^{4 \times 4} ; \boldsymbol{B}_{c l}=\left[\begin{array}{l}
0 \\
0 \\
1 \\
0
\end{array}\right] \in R^{4 x 1} ; \boldsymbol{C}_{c l}=\left[\begin{array}{llll}
0 & 1 & 0 & 0
\end{array}\right] \in R^{1 \times 4}
$$




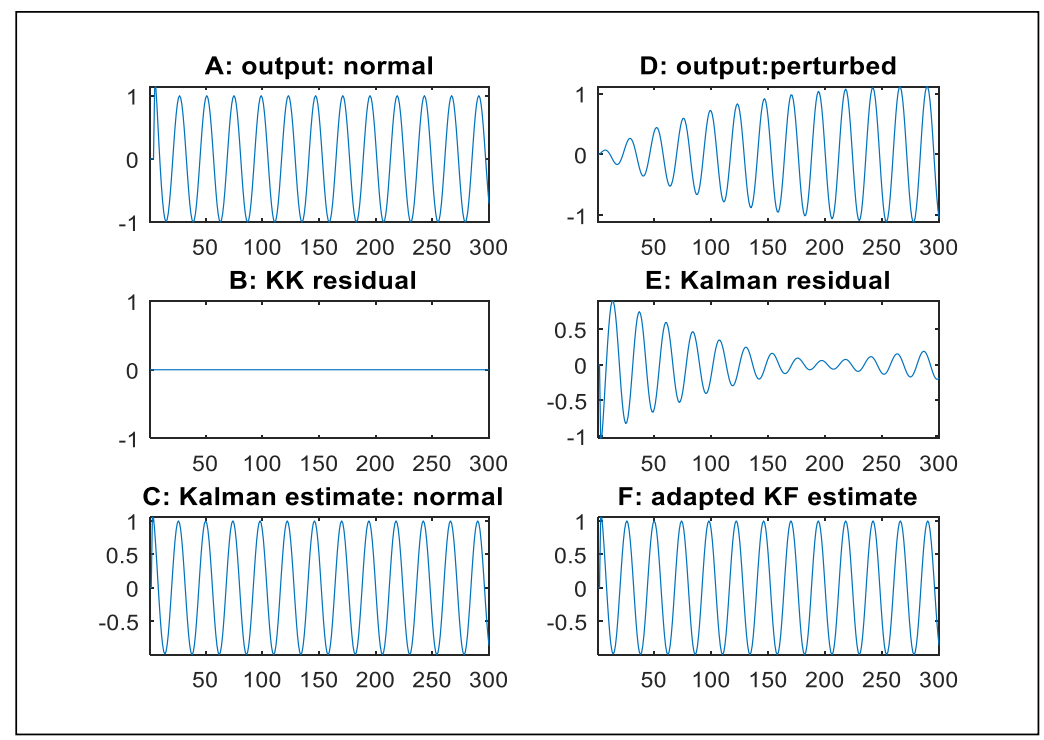

Fig. 2 nominal and perturbed signal: role and benefit of adaptation

Fig. 2 shows the performance of the robust combined feedforward-feedback controller using the online adaptive filter under model perturbation. Subfigures A, B, and C show the nominal unperturbed signal, KF residual which is negligibly small, and the KF estimate. Subfigures D, E and F show the perturbed signal, KF residual which is non-negligible and the Kalman filter estimate

\subsection{Comparison with Conventional Controllers}

The proposed scheme based on the KF estimate of the signal is significantly superior to the conventional approach in tracking a given reference despite the unknown stochastic disturbance and the zero-mean white measurement noise process. The signal buried in the output is estimated in order to develop the controllers without the need for the measurement of the disturbance and measurment noise. Subfirues of Fig. 2 clearly show the superior performance of the proposed scheme over the conventional one

The tracking error of the conventional scheme, even with the availability of the measusurement of the disturbance and measurement noise, is a random process. Tracking a desired trajectory in two-dimensional plane exhibit unwanted random deviations around the given desired trajectory. On the other hand, the proposed scheme provides a proven framework for autonomous vehicles to reliably and accurately track a given trajectory, all without the onerous requirement of the knowledge of the dusturbance and measurement noise statistics.

\subsection{Appplications: Autonomous Terrestal And Aerial Vehicles}

A vital application of the the proposed controller scheme would be for autonomous terrestrial and aerial vehicles to track a desired trajectory. Here, the controller may be designed and implemented by being inspired from the taining and testing schemes used with artificial neural network and in artificial intelligence [17-20].

Training phase: In the case of autonomous vehicle or drones, an experienced human operator is used for training. The human operator plays the role of the. The traing phase is conducted when the weather is 'good' with no snow and there is good visibility, as in this case, the desired trajectory is clearly seen, and disturbances such as wind gusts can be adequately modelled as zero-mean random processes. The input that drives the drone or the autonomous vehicle and the output, namely the navigational states such as position, velociry and acceleration, are all measured as a function of the location coordinates. The input and output data are then used to identify the system, the signal models and their associated KFs using the emulatorbased two-stage identification. Further, the model of the reference trajectory is determined and its internl model derived. Finally, the robust controller is designed. The training phase is successful if and only if the residual of the KF is a zero-mean white noise process and the estimate of the signal is the reference trajectory. 
Testing phase: In the testing phase, which is an autonomously-operational phase, the navigational states and the input are measured, and fed to the KFs. The combined feedforward-feedback is implemented using the estimated signal and identified signal model.

\section{Conclusions}

The KF-based The combined feedforward-feedback using the internal model principle is developed for a wide class of systems nonlinear systems approximated by piecewise Box-Jenkins model. The KF is shown to enjoy the key properties of estimating the signal, its model, the output error and the disturbance model even in low signal-to-noise ratio scenarios and with a spectral overlap between the disturbance and the signal. The robust stability and performance of the identified system, signal model, and controller is developed merely by adapting the KF residual without any time-consuming controller redesign. The proposed scheme has been shown to be underpinned by a rigorous mathematical foundation. A number of key properties of the KF have been derived and shown to hold if and only if the identified model embodied in the KF is identical to the system model. The striking feature of this novel controller is that there are no random deviations around the desired reference trajectory, even though the statistics (covariance) of the disturbance and the measurement noise were completely left out its design. Further, the performance is maintained even when there are model perturbations. With ample encouragement from this initial study, work is currently underway to develop a quadrotor that can provide autonomous help for real-life drones to track a specified trajectory reliably and accurately, despite wind drafts, by using the proposed feedforward-feedback control strategy.

\section{Acknowledgement}

The first author acknowledges the help of Professors C. P. Diduch and Maryhelen Stevenson of the University of New Brunswick

\section{References}

[1] Antsaklis, P, Editorial, Control Systems and the Quest for Autonomy IEEE transactions on automatic control, vol. 62, no. 3, march 2017

[2] Mohamed Fanni and Ahmed Khalifa, (2017),“A New 6-DOF Quadrotor Manipulation System: Design, Kinematics, Dynamics, and Control” IEEE/ASME Transactions On Mechatronics, VOL. 22, NO. 3, pp; 1315-1326

[3] Xiaodong Zhang, Xiaoli Li, Kang Wang, and Yanjun Lu,(2014), “A Survey of Modelling and Identification of Quadrotor Robot”, Hindawi Publishing Corporation, ID 320526, http://dx.doi.org/10.1155/2014/320526

[4] T.Soderstrom,(2006),"System Identification for the Errors in-Variables Problem", Transactions of the Institute of Measurement and Control 34(7) 780-792

[5] Stephane Thil, et.,al., (2009), "Unifying some higher-order statistic-based methods for errors-in-variables model identification", Automatica 45, 8, DOI:10.1016

[6] Guest Editorial: Emerging Trends in LPV-Based Control of Intelligent Automotive Systems, Vol. 14, Issue 18, 17 December 2020, IET Control Theory \& Applications

[7] D. Abbott (2014), "Applied Predictive Analytics: Principles and Techniques for the Professional Data Analyst”, John Wiley \& Sons

[8] G. J. Mallory and R. Doraiswami (1999), “A Filter for On-line Estimation of Spectral Content, IEEE Transactions on Instrumentation and Measurement”, Vol. 48, No.6, pp. 1047-1055

[9] Francis, Bruce, F, and Murry, W. Wonham. 1976. "The Internal Model Principle of Control Theory." Automatica 12: $457-465$

[10] Doraiswami, R and Cheded. L (2017), Robust Fault-Tolerant Control Using an Accurate Emulator-Based Identification Technique, International Journal of Control, ISSN: 0020-7179, 1336-5820 (online) Journal homepage: http://www.tandfonline.com/loi/tcon20 http://dx.doi.org/10.1080/00207179.2017.1318452

[11] R. Doraiswami and L. Cheded (2018), "Robust Kalman Filter-Based Least Squares Identification of a Multi Variable System", IET Control Theory and Applications; The Institution of Engineering and Technology, ISSN 1751-8644, www.ietdl.org 